\title{
ESTRUCTURA, DISTRIBUCIÓN Y ESTADO DE CONSERVACIÓN DE LOS BOSQUes de Prosopis fLEXUOSA DEL Bolsón de Fiambalá (CATAMARCA)
}

\author{
JUAN A. ALVAREZ ${ }^{1,2}$, PABLO E. VILLAGRA' ${ }^{1,2}$, ERICA M. CESCA ${ }^{1}$, FACUNDO ROJAS $^{1}$ y \\ SILVIA DELGADO ${ }^{1}$
}

\begin{abstract}
Resumen: Los bosques de Prosopis flexuosa han permitido históricamente la subsistencia y desarrollo de los oasis productivos al pie de la Cordillera de Los Andes, mediante el uso de sus recursos forestales. La normativa actual determina que la utilización de estos recursos dependa del ordenamiento territorial de los bosques. Sin embargo, el conocimiento de los bosques de zonas áridas es incompleto. Nos propusimos determinar: distribución, estructura actual, estado de conservación y crecimiento leñoso de los algarrobales del Bolsón de Fiambalá. Se identificaron unidades boscosas mediante la clasificación supervisada de imágenes. Se realizaron estudios poblacionales de $P$. flexuosa, se midieron variables alométricas y se aplicaron técnicas dendrocronológicas tradicionales. Se distinguieron dos tipos de bosque: denso con mayor cobertura total (39\%) y ralo $(21 \%)$. Las estructuras de diámetro fueron similares, sin embargo fue mayor la altura total, la cantidad de árboles maderables y el área basal en el bosque denso. Fue similar el grado de utilización y el estado de conservación. Las diferencias entre los bosques pueden explicarse por la posición del bosque relativa al acuífero subterráneo y al tipo de suelo. La conservación y utilización controlada de estos recursos, constituye un aporte a la mitigación del cambio climático ya que los algarrobales constituyen el principal reservorio de $\mathrm{C}$ en los sistemas desérticos.
\end{abstract}

Palabras clave: Bosque abierto, desierto del Monte, productos maderables, atributos poblacionales.

Summary: Structure, distribution and conservation status of the Prosopis flexuosa woodlands in the Bolsón de Fiambalá (Catamarca). The Prosopis flexuosa woodlands have historically allowed the survival and development of productive oasis at the foot of the Andes mountains, through the use of their forest resources. Current regulations state that the use of these resources depends on the zoning of forests. However, knowledge of dryland forests is incomplete. Our aim were to determine the distribution, structure, preservation and growth of these woodlands. Woodland units were identified by supervised classification of images. Population studies of $P$. flexuosa were performed allometric variables were measured and applied traditional dendrochronological techniques. Two woodland types were distinguished: dense forest with greatest total coverage $(39 \%)$ and sparse forest $(21 \%)$. Basal diameter structures were similar, however, in the dense forest were greater overall height, the amount of timber trees and basal area. In both forests, was similarly use and conservation status. The differences between forests can be explained by the position of the forest on the underground aquifer and soil type. The conservation and controlled use of these resources is a contribution to mitigating climate change because Prosopis woodlands are the main reservoir of $\mathrm{C}$ in desert.

Key words: Woodland, Monte desert, wood products, population attributes.

\footnotetext{
${ }^{1}$ Departamento de Dendrocronología e Historia Ambiental, IANIGLA-CONICET-CCT Mendoza. Av. Ruiz Leal s/n, Parque General San Martín, CP 5500, cc 330. Mendoza, Argentina. * jalvarez@mendoza-conicet.gov.ar.

${ }^{2}$ Facultad de Ciencias Agrarias, Universidad Nacional de Cuyo, Almirante Brown 500, CP 5505, Chacras de Coria, Mendoza, Argentina.
} 


\section{INTRODUCCIÓN}

Los ecosistemas áridos ocupan grandes extensiones pobladas principalmente con especies leñosas de gran exploración radical, por ende de considerable biomasa, que son considerados importantes reservorios de carbono (Roig, 1991; Kalaba et al., 2013). Estas regiones ocupan aproximadamente el $41 \%$ de la superficie terrestre (más de 6 mil millones de ha), de los cuales a los bosques nativos le corresponden el $18 \%$ de estas tierras áridas (FAO, 2013). En Argentina, los bosques existentes en ecosistemas desérticos son dominados por especies del género Prosopis, llamados comúnmente "algarrobos" (Alvarez \& Villagra, 2009). En la Provincia Biogeográfica del Monte, que ocupa $460.000 \mathrm{~km}^{2}$ desde Salta (al este de la cordillera de Los Andes) hasta la costa atlántica en Chubut, las poblaciones humanas han habitado desde tiempos antiguos los algarrobales, dependiendo de sus recursos naturales para su subsistencia y desarrollo. Las plantas leñosas, particularmente Prosopis flexuosa, se han utilizado en estos desiertos en gran medida, desde principios del siglo XX, permitiendo la expansión de los ferrocarriles, el desarrollo de la industria vitivinícola y otras actividades productivas en los oasis de riego (Abraham \& Prieto, 1999; Villagra et al., 2009). Las condiciones de extrema aridez y la historia de uso han determinado la transformación de la mayoría de estos algarrobales, siendo avanzado el estado de degradación actual de los mismos (Villagra et al., 2009). Actualmente en Argentina, los bosques nativos están protegidos por leyes nacionales y regionales, y la extracción de madera se realiza exclusivamente mediante planes de manejo permitidos. En este contexto, la gestión de los recursos forestales, comprende el ordenamiento territorial y el uso de prácticas silvícolas para la conservación y desarrollo de estos ecosistemas boscosos (FAO, 2001; LN 26331). Es necesario generar información que permita el manejo sustentable de estos bosques y planificar la restauración de estos ecosistemas (Newton \& Tejedor, 2011; Blackman, 2013).

Debido al bajo potencial de producción de madera, históricamente, los bosques de zonas áridas han sido descuidados en cuanto a su manejo forestal (FAO, 1999). Actualmente, la mayoría de los países han promulgado nuevas leyes y/o políticas tendientes a la conservación de los mismos, que tienen en cuenta en su formulación los principios básicos tendientes a la ordenación forestal sostenible (FAO, 2007). Los datos aportados por los inventarios forestales son indispensables para el conocimiento cualitativo y cuantitativo del recurso, constituyendo el fundamento de políticas idóneas de manejo y ordenación forestal sostenible (SAyDS, 2005; FAO, 1999).

El manejo adecuado de un área boscosa debe basarse en su estructura forestal (inventario) y en datos de productividad obtenidos a través de relaciones alométricas y el uso de métodos dendrocronológicos y silvícolas (Alder, 1980; Alvarez et al., 2011). La mayoría de las especies del género Prosopis poseen anillos de crecimiento de definición anual, lo que hace posible el estudio de la tasa de crecimiento radial por medio de la dendrocronología (Castro, 1994; Villalba et al., 2000). La ley de bosques determina que las provincias realicen el ordenamiento de los bosques basados en este tipo de inventarios y datos de crecimiento, sin embargo, el conocimiento de la estructura y distribución de los bosques de zonas áridas es incompleto.

Para el desierto del Monte, la descripción actual de los algarrobales (Alvarez et al., 2006; Cesca, 2014; Gil, 2014; Villagra et al., 2004), su historia de uso (Abraham \& Prieto, 1999; Prieto et al., 2003; Rojas, 2013), la potencialidad de manejo de los mismos (Alvarez et al., 2011a, 2011b, 2013) y las consecuencias del uso humano sobre estos recursos naturales (Meglioli et al., 2013; Vázquez et al., 2011), conforman información de base que es necesario seguir completando. En el Monte septentrional, las principales unidades boscosas se ubican en el fondo de valles y bolsones, en sitios donde los niveles freáticos son accesibles a las raíces de especies del género Prosopis (Rundel et al., 2006). El Bolsón de Fiambalá se encuentra en el oeste de la Provincia de Catamarca y el mapa de distribución potencial de estos bosques predice para la zona una alta probabilidad de existencia de bosques (Perosa et al., 2014). Además, algunos autores mencionan la existencia de bosques entre los médanos del valle (Burkart, 1976; Rojas, 2013). Nuestros objetivos fueron determinar la distribución y estructura actual, el estado de conservación y el crecimiento leñoso de los ecosistemas boscosos del Bolsón de Fiambala (Catamarca), y contribuir a 
la determinación de la potencialidad de manejo de estos algarrobales.

\section{MATERIALES Y MÉTOdos}

Área y especie de estudio

El área de estudio se ubicó en el Bolsón de Fiambalá, en el norte del desierto del Monte, oeste central de Catamarca, Argentina $\left(27-28^{\circ} \mathrm{S}, 67^{\circ} \mathrm{W}\right.$; 1400-1600 m.s.n.m.) (Fig. 1). En este trabajo se referirán como sinónimos los términos valle o bolsón. Las principales diferencias en su uso, se deben a cuestiones culturales por las cuales ciertos "valles", se han denominado históricamente "bolsones". Este valle o bolsón cubre una superficie del orden de los $2.000 \mathrm{~km}^{2}$, es ancho en su parte media $(30 \mathrm{~km})$, latitud de Saujil, al Norte de Fiambalá y es angosto hacia el Sur de El Puesto (2 km) (http://insugeo.org.ar/libros/cg_13/47_tineo. htm).

Como otros valles de la región, ocupa un área estrecha entre cadenas montañosas. Presenta una zona central plana, flanqueada por pendientes suaves de material aluvial y laderas erosionadas (Rundel et al., 2007). En esta región del Monte los suelos son principalmente Entisoles (INTA/CIRN, 1990). El clima es árido, con una temperatura media anual de $16,6^{\circ} \mathrm{C}$, con una media del mes más cálido de entre $23-24{ }^{\circ} \mathrm{C}$ y del mes más frío entre $7-8{ }^{\circ} \mathrm{C}$ (Bianchi \& Cravero, 2010). La precipitación media anual registrada en la estación meteorológica más cercana es de $173 \mathrm{~mm}$ concentrada principalmente en verano (Diciembre a Marzo) (Tinogasta, al sur del área de estudio (Fig. 1)) (Le Houérou, 1999).

En el Monte, P. flexuosa forma bosques abiertos en llanuras donde existe disponibilidad de agua en niveles subsuperficiales o en las galerías de los ríos (Alvarez \& Villagra, 2009). Como otras especies de Prosopis, esta leguminosa produce el enriquecimiento del suelo por medio de la fijación de nitrógeno y brinda importantes recursos a los pobladores locales (leña, forraje, sombra, entre otros) (Roig, 1993; Pasiecznik et al., 2001). Los frutos son consumidos por animales domésticos (en mayor proporción ganado vacuno, caprino), fauna nativa (roedores y aves) (Whitford, 2002) y muchas especies de invertebrados (Kingsolver et al., 1976).

La madera es altamente valorada por sus propiedades físicas, ya que es dura, pesada y densa debido al gran contenido de tanino (Castro, 1994). Además, posee un tejido leñoso complejo donde la presencia de parénquima terminal y vasos de mayor diámetro al comienzo de las bandas de crecimiento (porosidad semicircular), delimitan los anillos de crecimiento anual (Villalba, 1985), lo que permite conocer la edad de los árboles, su tasa de crecimiento anual y la capacidad de regeneración de la población (Kitzberger et al., 2000). Actualmente, en el Bolsón de Fiambalá, el uso de los bosques es silvopastoril, donde la leña es usada principalmente en cocción de alimentos y calefacción y se comercializa a mediana escala en otras ciudades de la región (Rojas, 2013). El área de estudio también es importante para la conservación de la biodiversidad, ya que alberga varios taxones endémicos o amenazados (Roig-Juñent et al., 2001).

Elección de las unidades de vegetación. Mapa de los algarrobales

Se procesaron imágenes satelitales Landsat 5 TM del 23 de marzo de 2005 y 7 ETM+ del 5 de febrero de 2000 (Path 232 Row 079). Cada imagen fue georreferenciada, y se utilizó la proyección en sistema de coordenadas POSGAR 1994, datum WGS 84, faja 2. Para identificar las distintas unidades boscosas de $P$. flexuosa, se realizaron ensayos con diversas clasificaciones de imágenes, obteniendo mejores resultados a partir de una clasificación supervisada a través del método de máxima verosimilitud, y de otra clasificación no supervisada realizada mediante el método $\mathrm{K}$-means. Los resultados se corrigieron y complementaron con salidas de campo y con el análisis de imágenes pancromáticas de alta resolución, del satélite CBERS 2B (sensor HRC) y del satélite QuickBird (Digital Globe) del 18 de agosto de 2010, ya procesadas y cargadas al servidor de mapas Google Earth (http://earth.google.com). En las visitas a campo, se realizó un análisis visual de la población de $P$. flexuosa, identificando cobertura, altura promedio y vegetación acompañante. Se registraron con GPS las coordenadas de cada zona identificada.

La definición de unidades de vegetación se realizó a partir de la clasificación y definición de categorías preexistentes para el Monte (Villagra et al., 2004; Alvarez et al., 2006; Cesca, 2014). De esta manera se buscó en las imágenes y en el trabajo de campo establecer unidades que respondieran a las 
siguientes categorías: áreas boscosas y no boscosas; definiendo el tipo de bosque de acuerdo a la cobertura de Prosopis (denso y ralo). Posteriormente se realizó un mapa síntesis, donde se determinaron 3 categorías (bosque denso, bosque ralo, oasis de riego y zona de ausencia de bosque), a partir de los mencionados métodos de clasificaciones supervisadas y no supervisadas (Fig. 1).

\section{Inventario de especies leñosas y caracterización edáfica}

Se eligieron al azar (aunque limitado por razones de accesibilidad en algunos casos), 24 sitios de muestreo distribuidos en ambos tipos de bosques, donde se distribuyeron parcelas de entre 0,1 y 0,4 de ha. El tamaño de las parcelas dependió de la densidad de árboles ya que se estableció como número mínimo de individuos muestreados por parcela: 40 (Cesca et al., 2014). Se muestrearon 12 parcelas en el Bosque Denso y 12 en el Bosque Ralo.

Para calcular la cobertura total de la comunidad, se analizaron imágenes de las parcelas de estudio ya procesadas y cargadas al servidor de mapas Google Earth (http://earth.google.com). Las imágenes fueron capturadas en formato TIFF para poder ser analizadas con el programa PC-Image (Scion Corporation), con una resolución de 429 píxeles de alto $\mathrm{x} 572$ píxeles de ancho. Por medio de la función "umbral", se obtuvo una imagen binaria (blanco y negro), facilitando el análisis mediante el programa PC-Image (Scion Corporation). Una vez delimitada el área de interés, se procedió a medir la superficie ocupada por las plantas en forma automática.

Al inicio de cada parcela se evaluó la comunidad de especies leñosas usando método fitosociológico (Braun Blanquet, 1979), empleando en cada caso cuadrados de 10 x $10 \mathrm{~m}$. Se registró la abundanciadominancia para las especies leñosas. No se registraron las especies del estrato herbáceo que fueron muy escasas en ambos tipos de bosques. La nomenclatura de las especies citadas sigue el Catálogo de Plantas Vasculares de la Argentina (Zuloaga \& Morrone, 1996; Zuloaga \& Morrone, 1999). En cada sitio se tomaron muestras de suelo, una bajo cobertura de P. flexuosa (BP) y otra en el área expuesta $(\mathrm{AE})$, estas últimas correspondieron a parches que no estuvieran bajo la cobertura de ninguna especie arbustiva o arbórea. Las muestras fueron colectadas a $20 \mathrm{~cm}$ de profundidad. Las variables analizadas y la metodología aplicada fue la siguiente: conductividad eléctrica: conductometría del extracto saturado (Richards, 1954), materia orgánica oxidable: método (Walkley, 1934), nitrógeno total $(\mathrm{N})$ método de Kjeldahl (Jackson, 1976), pH en pasta saturada (Richards, 1954), textura (internacional): método densimétrico de Boujoucos (Day, 1965), potasio intercambiable (K int) técnica de equilibrio con acetato de amonio $1 \mathrm{~N} \mathrm{pH} 7$ (Black et al., 1965). El contenido de calcio y magnesio se determinó a través del método complexométrico y el contenido de sodio por fotometría de llama (Jackson, 1976).

\section{Estructura poblacional de P. flexuosa}

En cada parcela se determinó el número de individuos adultos y juveniles (diámetro basal menor a 7,5 cm) (Villagra \& Villalba, 1999). Para todos los individuos de $P$. flexuosa se registró el número, altura y diámetro basal de cada fuste con cinta diamétrica o calibre digital, la altura total del árbol con hipsómetro digital Velmex III y las dimensiones de la copa (diámetro mayor y menor). Se calculó el área basal por hectárea para cada sitio de muestreo. Se clasificaron los individuos según el hábito de crecimiento en erectos, semierectos o decumbentes. Consideramos erectos (E) a los individuos que presentaron crecimiento vertical, semierectos (SE) a los individuos con un tallo principal ramificado y ramas sin tocar el suelo y decumbentes (D) aquellos árboles multitallares cuyas ramas tocan el suelo (Pasiecznik et al., 2001). El estado sanitario de los árboles se clasificó mediante una evaluación semicuantitativa: Sano (S), aquellos individuos con un $100 \%$ de ramas vivas; Medianamente sanos (MS), individuos con menos del $50 \%$ de ramas muertas; Sanidad regular (SR) más de $50 \%$ de ramas muertas e individuos con el $100 \%$ de ramas secas o árboles muertos en pie (M) (Alvarez et al., 2006). El potencial de uso de estos algarrobales fue evaluado estimando la cantidad de productos forestales presentes en cada individuo. Se determinó mediante un relevamiento no destructivo (Alvarez et al., 2006), la cantidad total de productos maderables presentes, teniendo en cuenta 5 tipos: a) vigas o maderas para aserrío: trozos mayores a 0,35 metros de diámetro y superiores a los 1,2 $\mathrm{m}$ de largo, b) rodrigón: entre $0,25 \mathrm{~m}$ y $0,35 \mathrm{~m}$ de diámetro y $2,5 \mathrm{~m}$ de largo, c) postes: entre $0,2 \mathrm{~m}$ y $0,25 \mathrm{~m}$ de diámetro y $2,5 \mathrm{~m}$ de 

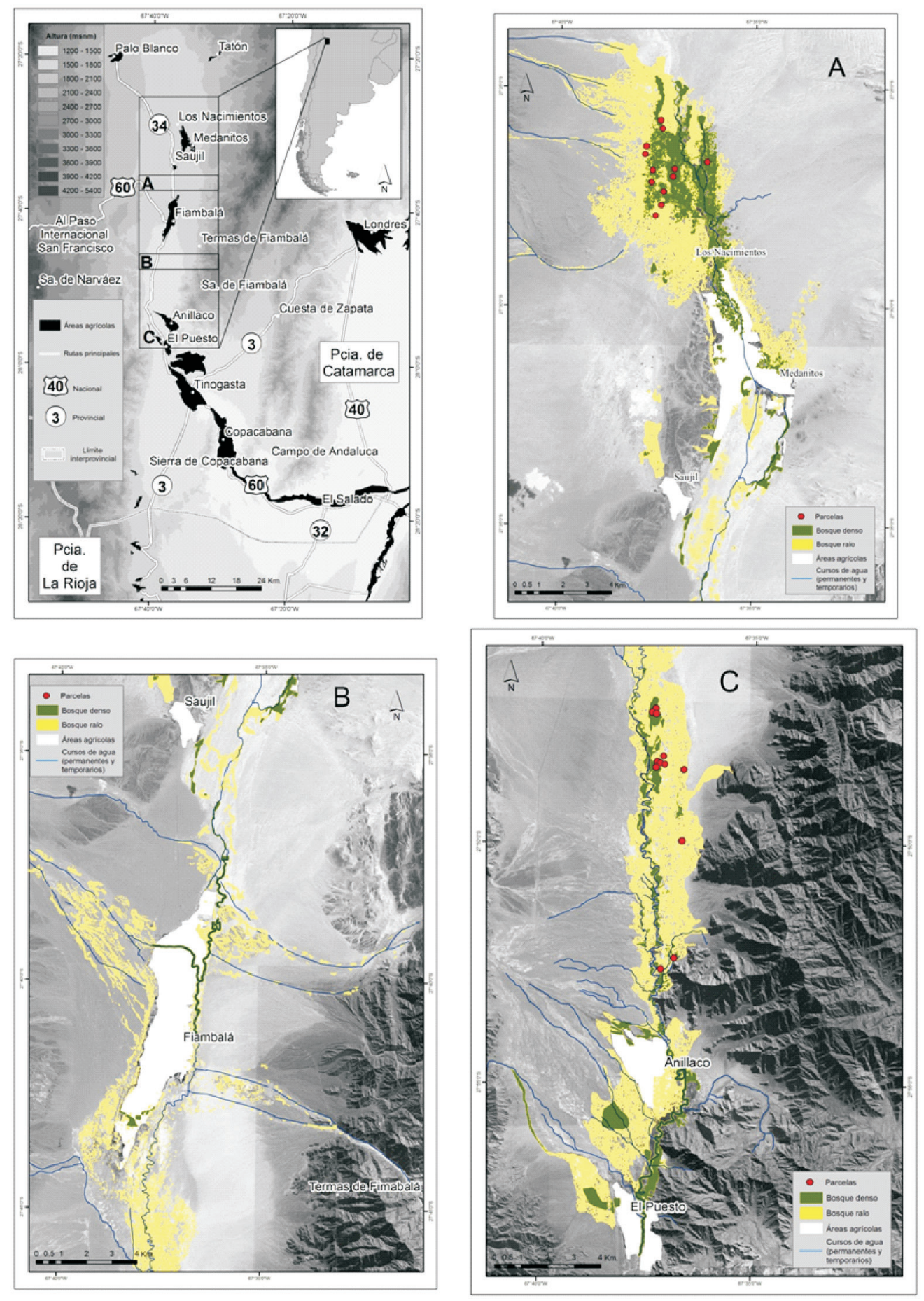

Fig. 1. Mapa de ubicación del área de estudio y distribución de los algarrobales del Bolsón de Fiambalá. A: Detalle de los algarrobales al norte del Bolsón. B: Zona central del Bolsón, que incluye el área urbana de Fiambalá y sus cultivos. C: Detalle de los de los algarrobales al sur del Bolsón. En escala de grises se representa la altura sobre el nivel del mar. Las áreas boscosas estudiadas se representan con verde al bosque denso y amarillo al bosque ralo. Los puntos rojos representan los sitios de muestreo. 
largo, d) $1 / 2$ postes: entre $0,2 \mathrm{~m}$ y $0,25 \mathrm{~m}$ y hasta 2 $\mathrm{m}$ de largo y e) parralero: hasta $0,2 \mathrm{~m}$ de diámetro y 2,5 $\mathrm{m}$ de largo.

\section{Incremento diamétrico anual del leño}

La presencia de anillos de crecimiento anual de esta especie nos permitió utilizar un enfoque dendroecológico para determinar las tasas de crecimiento (Villalba, 1985). Mediante motosierra, se tomaron muestras (secciones transversales) de árboles muertos en pie y tocones (restos de árboles adultos cortados a la altura de la base) de los algarrobales estudiados (sitio Los Nacimientos, Fig. 1B). Las muestras se pulieron y fecharon siguiendo métodos dendrocronológicos tradicionales (Stokes \& Smiley, 1968). Se estimó el incremento medio diamétrico anual en base a 19 árboles muestreados.

\section{Análisis de datos}

Se realizó una prueba $\mathrm{T}$ para evaluar, entre los bosques denso y ralo, diferencias en la cobertura total de la vegetación, área basal, densidad de árboles adultos y renovales, altura total, diámetro de copa, número de fustes, altura de fustes, bioforma, estado sanitario y productos maderables. La unidad muestreal considerada fue la parcela, por lo que las variables medidas en cada individuo fueron promediadas para esta superficie. En el caso de las proporciones los datos fueron transformados mediante la función arco seno de la raíz (xi/100) para cumplir con los supuestos de homogeneidad de varianza y normalidad (Zar, 1984). Para los datos del suelo se realizó un análisis de la varianza en el que se compararon los valores medios de acuerdo al tipo de bosque (BD y BS), micrositio (BP y AE) y la interacción de ambos (Zar, 1984).

\section{Resultados}

\section{Distribución de los algarrobales}

Se detectaron 12680 ha de bosques de $P$. flexuosa distribuidos en las zonas más bajas del Bolsón de Fiambalá (entre 1400 y 1600 m s.n.m.) (Fig. 1). De estos bosques, 2203 ha se presentan como bosque denso y 10477 ha al bosque ralo. El bosque denso tiene una mayor superficie en la parte norte del Bolsón, ocupando la zona más baja del mismo siendo rodeado por bosque ralo (Fig. 1A). En la región sur se encuentra una mayor extensión del bosque ralo encontrándose pequeñas manchas de bosque denso (Fig. 1B).

Inventario de especies leñosas y caracterización edáfica

El bosque denso presentó una mayor cobertura total $(39,26 \pm 15,48)$ que la del bosque ralo $(21,62 \pm 11,29)(\mathrm{T}=3,19 ; P=0,0042)$. En los dos tipos de bosques, la especie dominante fue $P$. flexuosa con $34,5 \%$ de cobertura media el bosque denso y $16.1 \%$ el bosque ralo. La comunidad de especies leñosas acompañantes fue muy similar en los dos sitios, siendo Capparis atamisquea y Suaeda divaricata las que mostraron mayor presencia y cobertura media. A su vez, estas dos especies presentaron mayor cobertura en el bosque denso que en el ralo (Tabla 1). Otras especies altamente presentes fueron Bulnesia retama y Prosopis argentina, que no mostraron diferencias entre ambos bosques, los que presentaron suelos areno - arcillosos.

Los suelos del bosque ralo fueron más salinos que los del bosque denso, tal como lo evidenció la mayor conductividad eléctrica y RAS, y el mayor contenido de $\mathrm{Mg}$ y $\mathrm{Ca}$. El contenido de materia orgánica, N, P y K fue mayor bajo la cobertura de Prosopis que en las áreas expuestas en los dos tipos de bosques (Tabla 2), observándose diferencias entre los tipos de bosques solo en el contenido de P.

\section{Estructura poblacional de P. flexuosa}

Los parámetros estructurales analizados mostraron mayor densidad de árboles adultos, área basal y cobertura total de P. flexuosa en el bosque denso que en el ralo (Tabla 3). Para un total de 668 árboles muestreados, las estructuras diamétricas de ambos bosques mostraron distribuciones relativamente similares (Fig. 2). Se observó un mayor número de individuos en todas las clases del bosque denso, excepto para la clase de 2,5 $\mathrm{cm}$ de diámetro, donde el bosque ralo presentó en promedio 120 árboles. ha ${ }^{-1}$ vs 75 registrados en el bosque denso. El bosque denso presentó un promedio mayor de altura de los árboles adultos y altura del fuste principal, aunque el diámetro de copa fue similar. Esto se relaciona con la bioforma o hábito de crecimiento, la proporción de árboles de diferentes categorías (E, SE y D) no presentó diferencias significativas entre los algarrobales.

Desde el punto de vista del manejo, en el bosque denso, hay una mayor la cantidad de árboles 
Tabla 1. Superficie total de cada bosque, Cobertura total de la vegetación, Cuadro sintético comparativo de los relevamientos de vegetación. Los valores expresados en números romanos indican los grados de presencia (V=> 80\%; IV=60-80\%; III= 40-60\%; II= 20-40\%; I=<20\%), seguido de los valores cobertura promedio.

\begin{tabular}{|c|c|c|}
\hline & Bosque Denso & Bosque Ralo \\
\hline Superficie total (ha) & 2203 & 10477 \\
\hline \multicolumn{2}{|l|}{ Cobertura total } & $21,6(11,29)$ * \\
\hline \multicolumn{3}{|l|}{ Especies } \\
\hline Prosopis flexuosa & V 34,5 & V 16,08 \\
\hline Capparis atamisquea & V 10,6 & V 2,25 \\
\hline Suaeda divaricata & III 7,09 & V 1,63 \\
\hline Atriplex lithophila & IV 1,27 & III 2,5 \\
\hline Bulnesia retama & IV 0,45 & II $\quad 0,46$ \\
\hline Prosopis argentina & II $\quad 0,05$ & I 1,08 \\
\hline Geoffroea decorticans & I $\quad 0,05$ & I 0,08 \\
\hline Atriplex lampa & I $\quad 0,09$ & I $\quad 0,04$ \\
\hline Baccharis salicifolia & . & I 0,08 \\
\hline Larrea cuneifolia & . & I 0,04 \\
\hline Larrea divaricata & I 0,09 & . \\
\hline Cercidium praecox & I 0,05 & . . \\
\hline
\end{tabular}

${ }^{*} \mathrm{~N}=24, \mathrm{~T}=3,19, \mathrm{p}=0,0042$

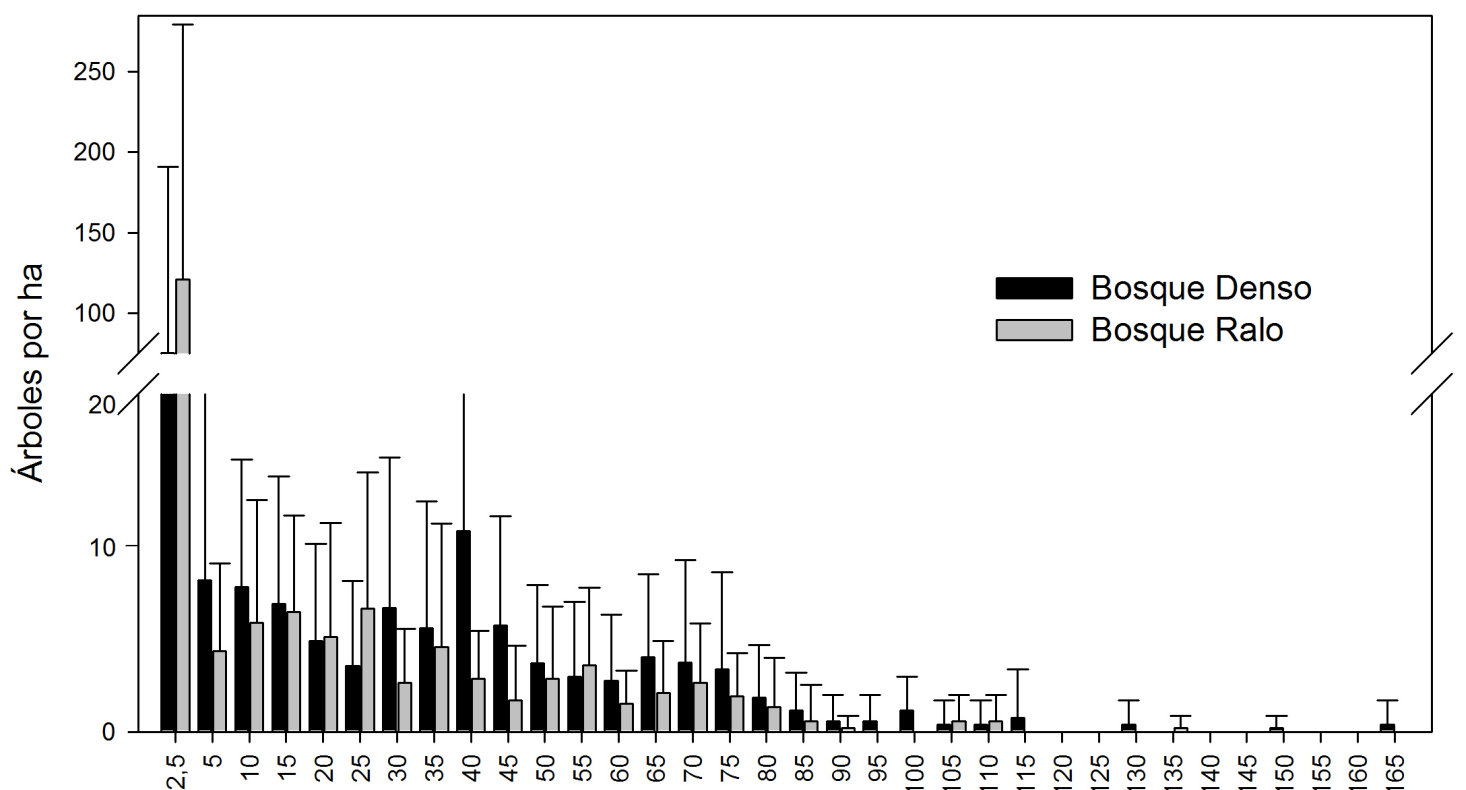

Diámetro basal equivalente $(\mathrm{cm})$

Fig. 2. Distribución de frecuencias de los diámetros basales de los algarrobales del Bolsón de Fiambalá. Las barras verticales representan los valores medios en clases de $10 \mathrm{~cm}(5 \mathrm{~cm}$ para los $<10 \mathrm{~cm})$ y las líneas verticales el desvío estándar. Los sitios de muestreo fueron 12 en cada tipo de bosque. 
Bol. Soc. Argent. Bot. 50 (2) 2015

Tabla 2. Características físico-químicas de los suelos de los bosques de Fiambalá. Los valores

representan la media (desvío estándar). También se indican los resultados del ANAVA para cada tipo de bosque, los valores en negrita representan diferencias significativas $(P<0,05)$. El tipo de Micrositio se indica como bajo Prosopis (BP) y área expuesta (AE). Los tipos de suelo se indican como: Arenoso= Are, Arcilloso= Arc y Limoso= Lim.

\begin{tabular}{|c|c|c|c|c|c|c|}
\hline \multirow{2}{*}{ Variable } & \multirow{2}{*}{ Micrositio } & \multirow{2}{*}{ Bosque Denso } & \multirow{2}{*}{ Bosque Ralo } & \multirow{2}{*}{ Tratamiento } & \multirow{2}{*}{ Micrositio } & \multirow{2}{*}{$\begin{array}{l}\text { Interacción } \\
\text { Trat. }{ }^{*} \text { Micr. }\end{array}$} \\
\hline & & & & & & \\
\hline \multirow{2}{*}{ Textura } & $\mathrm{BP}$ & Are-Arc-Lim & Are-Arc & \multirow{2}{*}{$\cdots$} & \multirow[b]{2}{*}{$\cdots$} & \multirow[t]{2}{*}{ 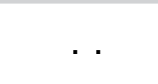 } \\
\hline & $\mathrm{AE}$ & Are-Arc & Are-Arc & & & \\
\hline \multirow{4}{*}{$\begin{array}{c}\mathrm{CE} \\
\left(\mathrm{mS} / \mathrm{cm} \mathrm{a} 25^{\circ} \mathrm{C}\right)\end{array}$} & & 8006,89 & 17962,86 & \multirow{2}{*}{$F=4,88$} & \multirow{2}{*}{$F=0,92$} & \multirow{2}{*}{$F=0,57$} \\
\hline & $\mathrm{BP}$ & $-3220,9$ & $-16356,79$ & & & \\
\hline & & 7401,67 & 29257,86 & \multirow{2}{*}{$P=0,03$} & \multirow{2}{*}{$P=0,01$} & \multirow{2}{*}{$P=0,45$} \\
\hline & $\mathrm{AE}$ & $-8236,19$ & $-32968,41$ & & & \\
\hline \multirow{4}{*}{$\mathrm{pH}$} & & 7,1 & 7,09 & \multirow{2}{*}{$F=0,001$} & \multirow{2}{*}{$F=0,18$} & \multirow{2}{*}{$F=0,23$} \\
\hline & $\mathrm{BP}$ & $-0,04$ & $-0,03$ & & & \\
\hline & & 7,1 & 7,1 & \multirow{2}{*}{$P=0,97$} & \multirow{2}{*}{$P=0,67$} & \\
\hline & $A E$ & $-0,06$ & $-0,06$ & & & $P=0,63$ \\
\hline & & 44,39 & 85,71 & & & \\
\hline Calcio & $\mathrm{BP}$ & $-22,2$ & $-68,11$ & $F=5,23$ & $r=0,02$ & $r=0,0001$ \\
\hline (meq/l) & & 40,78 & 83,91 & & & \\
\hline & $\mathrm{AE}$ & $-42,11$ & $-69,82$ & $P=0,03$ & $P=0,87$ & $P=0,96$ \\
\hline & & 16,61 & 37,43 & & & $F=0,01$ \\
\hline Magnesio & $\mathrm{BP}$ & $-6,2$ & $-36,45$ & $F=4,48$ & $r=0,11$ & $r-0,01$ \\
\hline$($ meq/l) & & 20,42 & 39,51 & & & \\
\hline & $A E$ & $-20,26$ & $-36,63$ & $P=0,04$ & $P=0,74$ & $P=0,92$ \\
\hline & & 19,22 & 54,93 & $F=3.26$ & $F=0.27$ & $F=1.12$ \\
\hline Sodio & BP & $-7,64$ & $-59,66$ & $r=3, \angle 0$ & $r=0,2 t$ & $r=1,12$ \\
\hline (meq/l) & & 17,13 & 167,87 & & & \\
\hline & $A E$ & $-21,91$ & $-234,07$ & $P=0,08$ & $P=0,6$ & $P=0,29$ \\
\hline & & 3,46 & 5,78 & & & \\
\hline RAS & $\mathrm{BP}$ & $-1,05$ & $-4,74$ & $F=4,18$ & $F=0,04$ & $F=2,11$ \\
\hline RAS & & 2,64 & 16,32 & & & \\
\hline & $\mathrm{AE}$ & $-2,17$ & $-20,84$ & $P=0,05$ & $P=0,85$ & $P=0,15$ \\
\hline & & 1543,11 & 852 & $F=316$ & $F=17.31$ & $F=2,47$ \\
\hline Nitrógeno & $\mathrm{BP}$ & $-467,79$ & $-265,56$ & $r=3,16$ & $r=17,31$ & $r=2,4 r$ \\
\hline$\left(\mu \mathrm{g} \cdot \mathrm{g}^{-1}\right)$ & & 640 & 678 & & & \\
\hline & $\mathrm{AE}$ & $-338,45$ & $-504,25$ & $P=0,08$ & $P=0,0003$ & $P=0,12$ \\
\hline & & 35,72 & 18,77 & & $F=785$ & $F=192$ \\
\hline Fósforo & BP & $-12,23$ & $-8,52$ & $F=1,53$ & $F=1,85$ & $r=1,92$ \\
\hline$\left(\mu \mathrm{g} \cdot \mathrm{g}^{-1}\right)$ & & 19,32 & 13,75 & & & \\
\hline & $\mathrm{AE}$ & $-13,05$ & -11 & $P=0,01$ & $P=0,009$ & $P=0,17$ \\
\hline & & 1529,4 & 1493,11 & $F=0,12$ & & $F=0,64$ \\
\hline Potasio & BP & $-683,94$ & $-1145,71$ & $r=0,12$ & $r=0,04$ & $r-0,04$ \\
\hline$\left(\mu g \cdot g^{-1}\right)$ & & 737,69 & 1283,17 & & & \\
\hline & $\mathrm{AE}$ & $-478,94$ & $-1279,14$ & $P=0,73$ & $P=0,02$ & $P=0,42$ \\
\hline & & 18,9 & 10,1 & $F=0.06$ & $F=8.19$ & $F=4.12$ \\
\hline MO & BP & $-14,1$ & $-5,4$ & $r=0,00$ & & \\
\hline$\left(\mathrm{mg} \cdot \mathrm{g}^{-1}\right)$ & & 4,9 & 14,8 & & & \\
\hline & $A E$ & $-3,2$ & $-19,3$ & $P=0,8$ & $P=0,007$ & $P=0,052$ \\
\hline
\end{tabular}




\section{J. A. Alvarez et al. - Bosques de Prosopis flexuosa de Catamarca}

maderables (>35 cm de Dab) (Fig. 2). Por su parte, el estado sanitario para todas las categorías consideradas presentó valores similares en ambos bosques (Tabla 3). En los bosques considerados, la proporción de individuos muertos en pie fue menor al $4 \%$. (Tabla 3 ). El número de tocones por ha medido en ambos bosques fue en promedio de 54,8 (entre 0 - 155) para el denso y 35 (entre 0 - 125) para el ralo, no registrando estos valores diferencias significativas (Tabla 3). La relación entre el área basal extraída (representada por los tocones) y el área basal total en pie fue del $15 \%$ en el bosque denso y del 9,3\% en el bosque ralo, existiendo sitios en ambos bosques donde no registramos presencia de tocones. El incremento diamétrico anual promedio de las muestras analizadas fue de $4,98 \mathrm{~mm}$, con un valor máximo registrado de 6,22 mm entre los 11 y los 25 años (Fig. 3).

La cantidad de productos forestales registrados en este estudio mostraron una gran disponibilidad en ambos bosques, observándose en todas las categorías valores mayores en el bosque denso que en el ralo, aunque las diferencias fueron marginalmente significativas $(P=0,07)$ (Tabla 3$)$. Es alta la variabilidad encontrada en los diferentes sitios, para una media total de productos forestales en el bosque denso de 333,8 (33,3 - 965) y en el ralo $131,4(20-392,5)$ (Fig. 4).

Tabla 3. Parámetros estructurales de los bosques de Fiambalá. Resultados de la prueba T, los valores en negrita representan diferencias significativas.

\begin{tabular}{|c|c|c|c|c|}
\hline & Bosque Denso & Bosque Ralo & $\mathbf{T}$ & $\boldsymbol{P}$ \\
\hline Área basal por ha $\left(\mathrm{m}^{2}\right)$ & $20,9(8,47)$ & $9,49(4,36)$ & 4,15 & 0,001 \\
\hline Densidad adultos (ind. ha-1) & $114,51(84,93)$ & $58,75(35,35)$ & 2,1 & 0,05 \\
\hline Densidad renovales (ind. ha-1) & $125,83(161,2)$ & $87,22(114,5)$ & $-0,68$ & 0,505 \\
\hline Altura total $(\mathrm{m})$ & $7,36(1,31)$ & $6,00(1,04)$ & 2,81 & 0,01 \\
\hline Diámetro copa adultos (m) & $8,35(1,12)$ & $8,38(1,98)$ & $-0,04$ & 0,968 \\
\hline Fustes $\left(\mathrm{N}^{\circ} . \mathrm{ha}^{-1}\right)$ & $364,51(205,7)$ & $266,67(162,1)$ & 1,29 & 0,209 \\
\hline Altura fustes promedio (m) & $1,34(0,44)$ & $1,01(0,27)$ & 2,17 & 0,04 \\
\hline Productos maderables $\left(\mathrm{N}^{\circ} . \mathrm{ha}^{-1}\right)$ & $331,32(333,89)$ & $131,4(121,2)$ & 1,95 & 0,071 \\
\hline Tocones $\left(\mathrm{N}^{\circ} . \mathrm{ha}^{-1}\right)$ & $54,8(44,5)$ & $35(39,17)$ & 1,16 & 0,258 \\
\hline \multicolumn{5}{|l|}{ Forma (\%) } \\
\hline Decumbente & $41(26)$ & $46(11)$ & $-0,61$ & 0,552 \\
\hline Semierecto & $40(20)$ & $44(11)$ & $-0,61$ & 0,551 \\
\hline Erecto & $19(15)$ & $10(7)$ & 1,88 & 0,077 \\
\hline \multicolumn{5}{|l|}{ Estado Sanitario (\%) } \\
\hline Sano & $50(20)$ & $63(17)$ & $-1,81$ & 0,084 \\
\hline Medianamente sano & $33(19)$ & $22(14)$ & 1,67 & 0,108 \\
\hline Sanidad regular & $13(11)$ & $11(9)$ & 0,46 & 0,651 \\
\hline Muerto & $4(6)$ & $3(5)$ & 0,33 & 0,744 \\
\hline
\end{tabular}


Bol. Soc. Argent. Bot. 50 (2) 2015

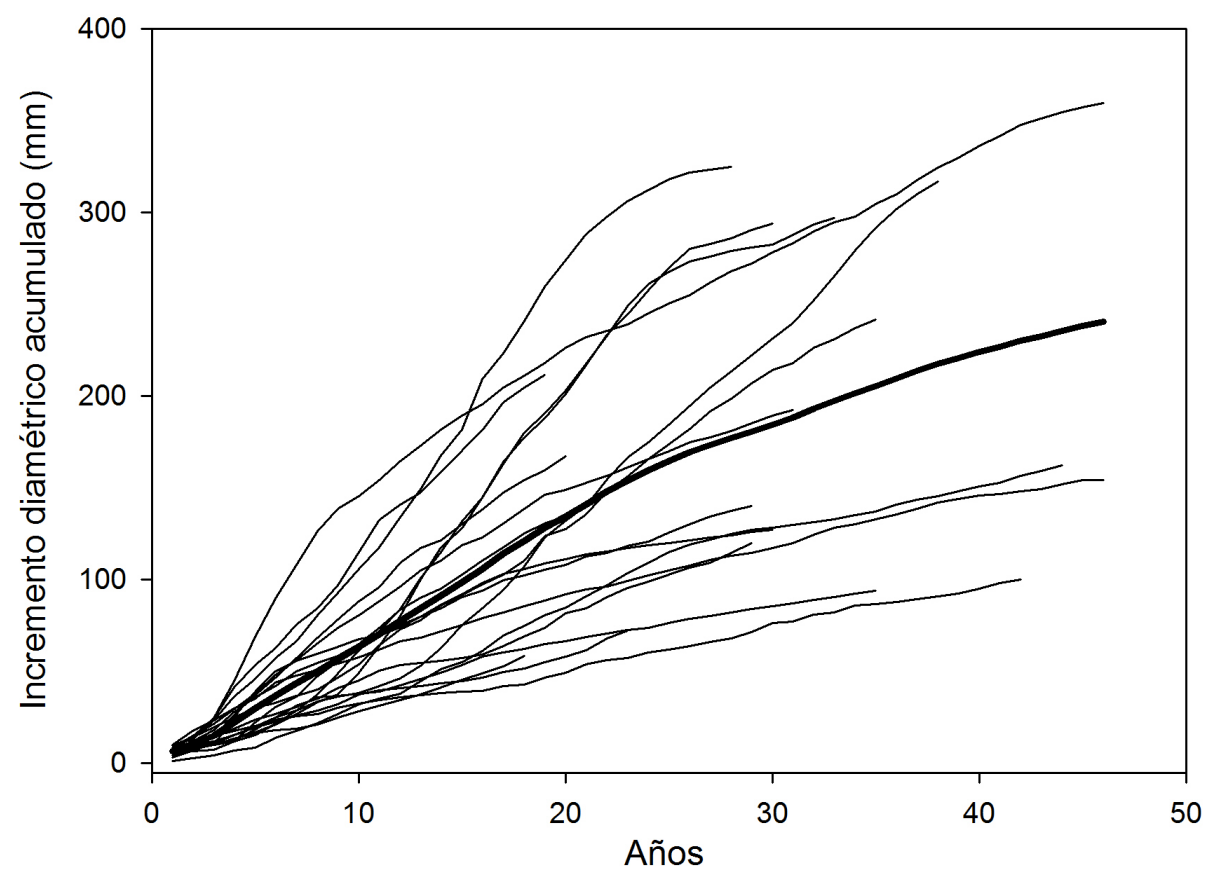

Fig. 3. Incremento diamétrico leñoso acumulado (mm). La línea gruesa indica el valor promedio de 19 árboles analizados. Las líneas finas representan las series individuales.

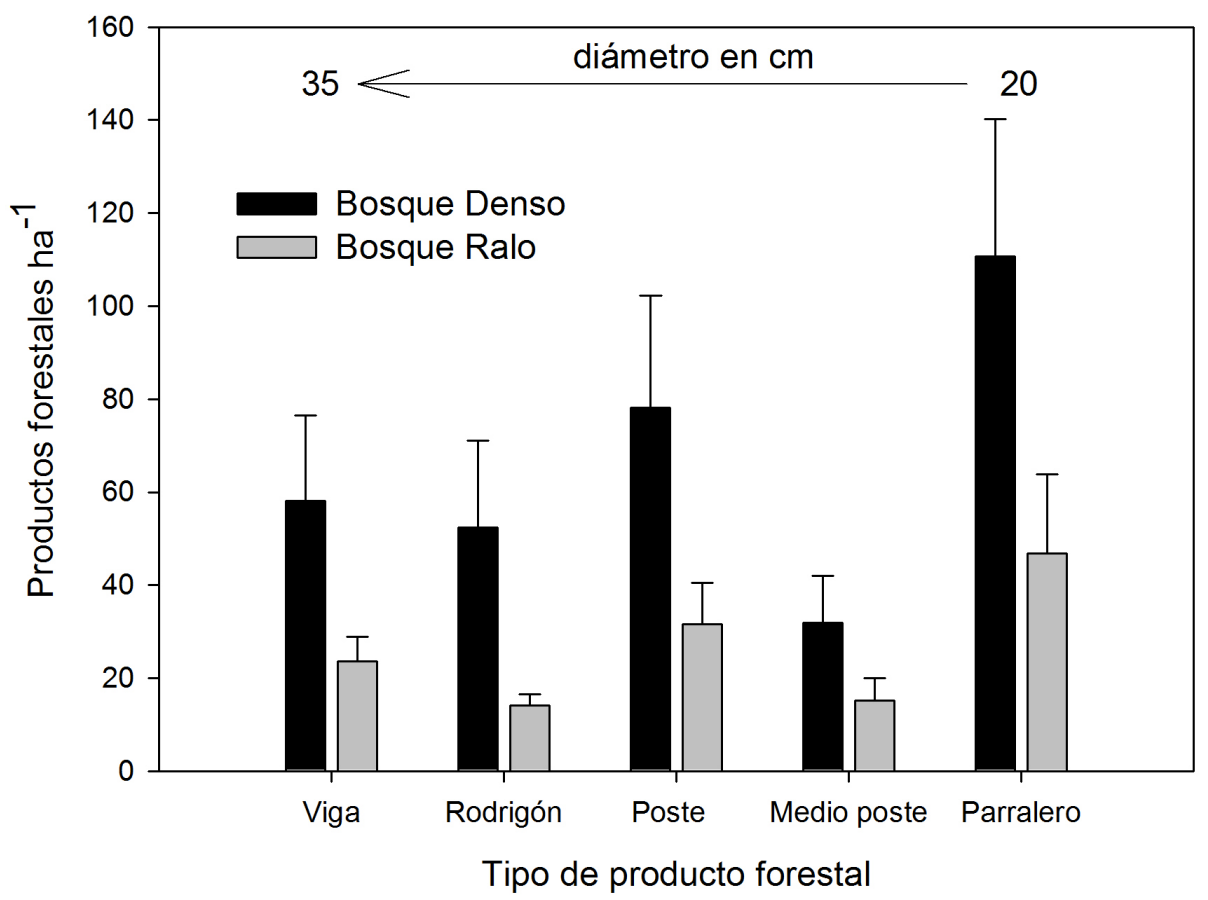

Fig. 4. Productos forestales registrados en ambos bosques. Las barras representan el valor medio y las líneas el error estándar. Los productos forestales están ordenados en forma decreciente con respecto al diámetro basal (parte superior del gráfico). La comparación entre bosques no presentó diferencias significativas en ninguno de los productos forestales evaluados. 


\section{Discusión}

Los bosques de P. flexuosa en el Bolsón de Fiambalá ocupan actualmente 12680 ha de la zona más baja de la cuenca. Este área se encuentra dentro de la zona de distribución potencial para los bosques propuesta por Perosa et al. (2014) a partir de modelado de nicho. Esta distribución podría estar asociada a la accesibilidad del agua freática que le permite a esta especie desarrollar formaciones boscosas en zonas con precipitaciones menores a $350 \mathrm{~mm}$ anuales (Jobbágy et al., 2011; Villagra et al., 2010). Sin embargo, se encuentran zonas donde la probabilidad de encontrar bosques de esta especie es muy alta (Perosa et al., 2014) donde actualmente no se encontraron formaciones boscosas. Esto podría deberse a factores aleatorios de dispersión, a otros factores limitantes o a una retracción del bosque producto del uso humano. La utilización histórica de estos bosques, se debe a la estrecha relación geográfica entre el bosque y las poblaciones humanas de la región, ya que los algarrobales estudiados se localizan en una importante zona del circuito productivo y turístico del noroeste argentino. Los poblados de Fiambalá, Medanitos, Saujil, Anillaco y El Puesto, y sus áreas irrigadas, se encuentran también en la zona baja del valle estudiado (Fig. 1), instalados sobre antiguos algarrobales, pudiendo observarse relictos de esta especie dentro de los pueblos. En general, los algarrobales del norte del Monte han registrado desde fines del siglo XIX, la utilización intensa de sus productos forestales (madera, postes, carbón y leña) (Villagra et al., 2009; Rojas, 2012). En este trabajo, encontramos que el área basal de los tocones encontrados representa el $15 \%$ con respecto al área basal total, lo que sugiere un uso importante si consideramos que en el área de estudio se encontraron valores de área basal total que están entre los más grandes del Monte (Villagra et al., 2004; 2005).

Los dos tipos de bosques detectados a través de la clasificación de imágenes satelitales resultaron ser diferentes en cobertura total, área basal, densidad de individuos adultos y cobertura de las especies leñosas dominantes; sin embargo, no se encontraron diferencias en la composición de especies. Tal como se ha observado en otros bosques del Monte, el área basal se relaciona directamente con la densidad de individuos adultos por hectárea (Alvarez et al., 2011a). Para los individuos adultos, otras variables importantes para el manejo forestal (altura total y altura de fuste) también fueron mayores en el bosque denso. Por el contrario, el diámetro de copa promedio fue similar en ambos bosques. Esto se relaciona con la bioforma o hábito de crecimiento, ya que la proporción de árboles de diferentes categorías (E, SE y D) no presentó diferencias significativas entre los algarrobales. Las diferencias entre ambos bosques pueden explicarse por estos aspectos: a- posición del bosque en la llanura eólica, donde el bosque denso se ubica en el fondo del valle intermedano y el ralo en las pendientes de los médanos (Gomez et al., En prensa); b- condiciones ambientales (tipo de suelo, disponibilidad de agua subsuperficial), más favorables en un caso que en el otro (Noy Meir, 1973; Villagra \& Cavagnaro, 2000), y c- diferencias en la intensidad de uso (Rojas, 2013; Bonino, 2006). Un aspecto a considerar es que por limitaciones logísticas de inaccesibilidad a los sitios más lejanos del bosque ralo, las diferencias en las coberturas de especies y estratos pueden haber sido levemente subestimadas, aunque no así las composiciones florísticas que parecen ser relativamente similares en todos los casos.

La mayor conductividad eléctrica, concentración de $\mathrm{Ca}, \mathrm{Mg}$ y RAS observada en el bosque ralo, sugiere condiciones más salinas que podrían limitar la productividad de $P$. flexuosa y otras leñosas dominantes reduciendo su crecimiento (Volkmar et al., 1998; Kovda et al., 1979). Por otro lado, se observó un mayor contenido de P y una incremento marginal en el $\mathrm{N}$ en el bosque denso. Si bien esto podría interpretarse como una mayor fertilidad, ésta podría ser consecuencia de la mayor cobertura de P. flexuosa, la especie que aporta mayor cantidad de materia orgánica en estos bosques (Alvarez et al., 2009). Esta idea es avalada por las diferencias encontradas entre los micrositios bajo P. flexuosa y áreas expuestas. Se observó mayor contenido de nutrientes bajo Prosopis que en el área expuesta (N, P y K), a la vez que un mayor contenido de MO registrado bajo Prosopis. Esto es coincidente con lo observado en otros desiertos del mundo (Pugnaire et al., 1996, Whitford, 2002) y en otros algarrobales del Monte (Cesca et al., 2012; Rossi et al., 2003; Abril et al., 2009; Miner et al., 2010). Durante los eventos puntuales de precipitación registrados en estas zonas (Noy Meir, 1973), en un corto tiempo los procesos bioquímicos adquieren gran 
importancia como formadores de estos suelos. Los suelos de áreas expuestas son en general más pobres en nutrientes que los suelos cubiertos por especies leñosas (Abril et al., 2009), la descomposición de la $\mathrm{MO}$ en estas regiones está asociada a fauna de descomponedores que son más abundantes bajo la copa de los algarrobos (Lagos, 2003). Podemos agregar que los valores de MO encontrados en los sitios BP del bosque denso se encuentran acordes con valores de otros sitios del Monte central como los algarrobales y jarillales de Ñacuñán (Asner et al., 2003, Rossi, 2004) y en el Monte patagónico, jarillales de Laguna Blanca (Rostagno \& del Valle, 1988), mientras que los sitios BP del bosque ralo comparten los valores más bajos de este tipo de ambientes junto con la comunidad de médanos de Ñacuñán (Asner et al., 2003).

Por su parte, no encontramos evidencias sobre diferencias en el manejo que puedan explicar las diferencias en la estructura de los bosques. No se observaron diferencias en el número de tocones por ha medido en ambos bosques, lo que podría interpretarse como una intensidad de uso similar en ambos tipos de bosques en los últimos años. Un aspecto a evaluar en futuros trabajos es la reconstrucción histórica del uso, teniendo en cuenta que la zona donde se encuentra el bosque denso se encuentra entre médanos altos que podrían haber impedido el acceso para su utilización, por lo que el grado de protección en tiempos históricos pueda haber sido mayor, como ha ocurrido en otras zonas donde los bosques se ubican entre médanos (Alvarez et al., 2006).

La descripción de la estructura y el estado de conservación, permite conocer la situación actual de los bosques en estudio e integra la base de cualquier proyecto de manejo. Los bosques estudiados se encuentran entre los de mayor área basal y densidad de árboles adultos de los encontrados en la provincia biogeográfica del Monte, y sus árboles se encuentran entre los que alcanzan mayor desarrollo y presentan mayor crecimiento diamétrico anual. En Fiambalá, la altura total media de ambos bosques se ubica por encima de los algarrobales del Monte central (entre 3,9 y 4,4 m) y por debajo de los de Pipanaco (8,8 m) (Villagra et al., 2005). La densidad de árboles adultos fue muy superior (entre 131-331 para Fiambalá vs 116 - 62 para Telteca y Ñacuñán respectivamente) (Villagra et al., 2005). La cantidad de fustes por árbol fue para el bosque denso 1,51 y 1,82 para el bosque ralo, siendo similares a los de Pipanaco $(1,2)$. En bosques del Monte central (2,76 para Telteca y 4,38 para Ñacuñan) y sureños de Mendoza (33,3 en General Alvear), la proporción de individuos multifustales es mayor que de árboles de un solo fuste, (Alvarez et al., 2006; Villagra et al., 2005; Cesca et al., 2014). Esto es importante para el manejo forestal, ya que la multifustalidad determina crecimiento diferencial en algarrobos multitallares (Decumbentes) vs algarrobos de un solo fuste (Erectos) (Alvarez et al., 2011a). Los valores de crecimiento diamétrico están por encima de los incrementos registrados en Telteca (4 mm) y Ñacuñan (2 mm) y son similares a los registrados en Pipanaco (Catamarca con $6 \mathrm{~mm}$ ) (Villagra et al., 2005). Como en otras poblaciones de esta especie, la variación intrapoblacional es elevada (Fig. 3) (Villagra et al., 2005; Alvarez et al., 2011).

El análisis de los distintas variables estructurales y de crecimiento realizado concuerda con la propuesta de un gradiente de crecimiento y desarrollo leñoso observado entre las poblaciones de algarrobo del Monte, desde Salta (Valles Calchaquíes) (> crecimiento) hasta los algarrobales de General Alvear en el sur mendocino ( $<$ crecimiento) (Gil, 2014; Villagra et al., 2005; Cesca, 2014; Alvarez et al., 2011a). Lo anterior puede responder a factores ambientales (períodos más extensos de la temporada de crecimiento al norte del Monte) y/o genéticos, (manifestado tanto en aspectos morfológicos como en el crecimiento) (Duff et al., 1994; Cony, 1996; Villagra et al., 2004).

Combinando el estado sanitario y la forma de los individuos, se pueden definir posibles usos de la madera. Los dos bosques estudiados presentaron un buen estado sanitario con más del $60 \%$ de los árboles sanos y menos del $3 \%$ de árboles muertos, valores que son similares a los observados en otros bosques del Monte (Alvarez et al., 2006) y que en Fiambalá representan madera de mayor valor forestal (vigas y mutrucos). La forma de los individuos no presentó diferencias entre categorías (E, SE, D), sin embargo casi el $40 \%$ fueron decumbentes en ambos bosques (la mayoría de gran tamaño), lo que implica varios productos forestales por individuo (postes y rodrigones).

La información disponible, compuesta por el mapa de bosques (Fig. 1), el análisis de la estructura forestal (Tabla 1) y el estado de conservación nos 
permite considerar que la categoría de conservación propuesta para estos bosques (Categoría I - Rojo) por la Subsecretaría del Ambiente de Catamarca (LP N5311/10) son pertinentes. Sin embargo creemos necesaria la incorporación de parte estos territorios al sistema de áreas protegidas provincial para aumentar el porcentaje del escaso territorio del bioma Monte protegido en esta provincia (http:// www.ambiente.gov.ar/?IdArticulo=5473).

La conservación y utilización controlada de la biomasa leñosa a través de planes de manejo (LN 24331/07), constituye un aporte hacia la mitigación del cambio climático ya que los algarrobales, así como otros bosques de zonas áridas y semiáridas constituyen el principal reservorio de $\mathrm{C}$ en los sistemas desérticos.

\section{Agradecimientos}

Los autores agradecen a G. Zalazar por la asistencia técnica en el trabajo de campo, M. Medero por los análisis de laboratorio, C.B. Passera por la identificación de material de herbario y a S. Monge y M. Quiroga por el estudio dendrocronológico. Este estudio fue financiado por el CONICET y la SECTyP de la UNCuyo.

\section{Biblografía}

ABRAHAM, E.M. \& M.R. PRIETO. 1999. Vitivinicultura y desertificación en Mendoza. In: GARCÍA MARTÍNEZ, B. (ed.), Estudios de historia y ambiente en América: Argentina, Bolivia, México, Paraguay, pp. 109-135. IPGH - Colegio de México, México.

ABRIL, A., P.E. VILlAGRA \& L. NOE. 2009. Spatiotemporal heterogeneity of soil fertility in the Central Monte desert (Argentina). J. Arid Environ. 73: 901-906.

ALDER, D. 1980. Estimación del volumen forestal y predicción del rendimiento. Estudio FAO: Montes. FAO, Roma.

ALVAREZ, J.A., P.E.VILlagRA, B.E. ROSSI \& E. CESCA. 2009. Spatial and temporal litterfall heterogeneity generated by woody species in the Central Monte desert. Plant Ecol. 205: 295-303.

ALVAREZ, J.A., P.E. VILLAGRA, M.A. CONY, E.M. CESCA, \& J.A. BONINSEGNA. 2006. Estructura y estado de conservación de los bosques de Prosopis flexuosa D.C. en el Noreste de Mendoza, Argentina. Rev. Chil. Hist. Nat. 79: 75-87.
ALVAREZ, J.A., P.E. VILLAGRA, R. VILLALBA, M.A. CONY \& M. ALBERTO. 2011a. Wood productivity of Prosopis flexuosa D.C. woodlands in the central Monte: influence of the population structure and tree growth habit. J. Arid Environ. 75: 7-13.

ALVAREZ, J.A., P.E. VILLAGRA \& R. VILLALBA. 2011b. Factors controlling deadwood availability and branch decay in two Prosopis woodlands in the Central Monte, Argentina. Forest Ecol. Manag. 262: 637-645.

ALVAREZ, J.A., P.E. VILLAGRA, R. VILLALBA \& G. DEBANDI. 2013. Effects of the pruning intensity and tree size on multi-stemmed Prosopis flexuosa trees in the Central Monte, Argentina. Forest Ecol. Manag. 310: 857-864

ALVAREZ, J.A. \& P.E. VILlAGRA. 2009. Prosopis flexuosa DC. (Fabaceae, Mimosoideae). Kurtziana 35: 49-63.

BIANCHI, A.R. \& S.A.C. CRAVERO. 2010. Atlas climático regional digital de la República Argentina, INTA, Centro Regional Salta-Jujuy.

BLACKMAN, A. 2013. Evaluation forest conservation policies in developing countries using remote sensing data: An introduction and practical guide. Forest Policy Econ. 34: 1-16.

BONINO, E.E. 2006. Changes in carbon pools associated with a land-use gradient in the Dry Chaco, Argentina. Forest Ecol. Manag. 223: 183-189.

BRAUN BLANQUET, J. 1979. Fitosociología. Blume, Madrid.

BURKART, A. 1976. A monograph of the genus Prosopis (Leguminosae subfam. Mimosoideae). J. Arnold Arboretum 57: 219-249; 450-455.

CESCA, E.M, P.E. VILLAGRA, C.B. PASSERA \& J.A. ALVAREZ. 2012. Effect of Prosopis flexuosa on fodder species and its importance to the pastoral management of woodlands in the Central Monte desert. Rev. Fac. Cienc. Agrar. 44: 211-223.

CESCA, E.M., P.E. VILLAGRA \& J.A. ALVAREZ. 2014. From forest to shrubland: Structural responses to different fire histories in Prosopis flexuosa woodland from the Central Monte desert (Argentina) J. Arid Environ. 110: 1-7.

CASTRO, M.A. 1994. Maderas Argentinas de Prosopis. Atlas Anatómico. Secretaría General de la Presidencia de la Nación, Buenos Aires.

CESCA, E. 2011. Influencia del fuego en la estructura $y$ dinámica de los algarrobales del sudeste de Mendoza, Tesis Doctoral. Universidad Nacional de Córdoba. Córdoba (Argentina).

CONY, M.A. 1996. Genetic variability in Prosopis flexuosa D. C., a native tree of the Monte phytogeographic province, Argentina. Forest Ecol. Manag. 87: 41-49. 
DAY, P.R. 1965. Particle fractionation and particle-size analysis. In: BLACK, C.A. (ed.), Methods of soil analysis. Part I: Agronomy 9: 545-567.

DUFF, A.B., J.M. MEYER, C. POLLOCK \& P. FELKER. 1994. Biomass production and diameter growth of nine half-sib families of mesquite (Prosopis glandulosa var. glandulosa) and a fast growing Prosopis alba half-sib family grown in Texas. Forest Ecol. Manag. 67: 257-266.

F.A.O. 1999. Situación de los bosques del mundo. F.A.O. Roma.

F.A.O. 2001. Situación de los bosques del mundo. F.A.O. Roma.

F.A.O. 2007. Situación de los bosques del mundo. F.A.O. Roma.

F.A.O. 2014. The state of the world's forest genetic resources. Commission on genetic resources for food and agriculture, food and agriculture organization of the United Nations. Roma.

F.A.O. 2013. Bosques de las zonas áridas: Bosques y actividades forestales en las zonas áridas. Disponible en: http:/www.fao.org/forestry/aridzone/es/ [Acceso: 20 Junio 2013].

GIL, A. 2014. Estructura forestal y estado de conservación de los bosques de Prosopis chilensis y Prosopis flexuosa (algarrobales) de la Depresión del Río Bermejo, noreste de San Juan. Tesina de grado. IRNR, FCA, UNCuyo. Mendoza

GOMEZ, M.L., J.N. ARANIBAR, R. WUILLOUD, C. RUBIO., D. MARTINEZ, D. SORIA, R. MONASTERIO, P. VILLAGRA \& S. GOIRÁN. En prensa. Hydrogeology and hidrogeochemical modeling in phreatic aquifer of NE Mendoza, Argentina. J. Iber. Geol.

INTA/CIRN, 1990. Atlas de suelos de la República Argentina. Escala 1:500.000 y 1:1.000.000. Secretaría de Agricultura Ganadería y Pesca. Proyecto PNUD ARG. 85/019, Buenos Aires.

JACKSON, M.L., 1976. Análisis químico del suelo. Ediciones Omega, Barcelona.

JOBBÁGY, E.G., M.D. NOSETTO, P.E. VILLAGRA \& R.B. JACKSON. 2011. Water subsidies from mountains to deserts: Their role sustaining groundwater-fed oases in a sandy landscape. Ecol. Appl. 21: 2367-2379

KALABA, F.K., C.H. QUINN, A.J. DOUGILL \& R. VINYA. 2013. Floristic composition, species diversity and carbon storage in charcoal and agriculture fallows and management implications in Miombo woodlands of Zambia. Forest Ecol. Manag. 304: 99-109.

KOVDA, V.A., E.M. SAMOILOVA, J.L. CHARLEY\& J.J. SKUJINS. 1979. Soil processes in arid lands. In: GOODALL D.W., R.A. PERRY \& K.M.W. HOWES (eds.), Arid-Land Ecosystems: Structure, Functioning, and Management, Volume 1, pp. 439-
470. International Biological Programme, Cambridge University Press, Cambridge, UK.

KINGSOLVER, J.M., C.D. JOHNSON, S.R. SWIER \& A.L. TERAN. 1977. Prosopis fruits as a resource for invertebrates. In: SIMPSON, B.B. (ed.), Mesquite. Its biology in two Desert Scrub Ecosystems, pp. 108122. U.S./ibp synthesis series 4. Dowden, Hutchinson \& Ross, Inc.

KITZBERGER, T., T. VEBLEN \& R.VILLALBA. 2000. Métodos dendrocronológicos y sus aplicaciones en estudios de dinámica de bosques templados de Sudamérica. In: ROIG, F.A.C. (ed.), Dendrocronología en América Latina, pp 17-78. EDIUNC, Mendoza.

LAGOS, S.J. 2003. Diversidad biológica de las comunidades de artrópodos epígeos en áreas pastoreadas y no pastoreadas del Monte. Tesis Doctoral, Universidad Nacional de Cuyo, Mendoza.

LE HOUÉROU, H.N. 1999. Estudios e Investigaciones ecológicas de las zonas áridas y semiáridas de Argentina. Informe Interno IADIZA, Mendoza.

LEY PROVINCIAL N ${ }^{\circ}$ 5311. 2010. Ley de ordenamiento ambiental y territorial del bosque nativo. Senado y Cámara de Diputados de la Provincia de Catamarca

LEY NACIONAL N 26331. 2009. Ley de Presupuestos Mínimos de Protección Ambiental de los Bosques Nativos. Boletín Oficial de la República Argentina. Buenos Aires, 25 de Diciembre de 2007.

MEGLIOLI, P.A., J.N. ARANIBAR, P.E. VILLAGRA, J.A. ALVAREZ \& E.G. JOBBÁGY. 2013. Livestock stations as foci of groundwater recharge and nitrate leaching in a sandy desert of the Central Monte. Ecohydrol. 7: 600-611.

MINER, A.J., J.A. ALVAREZ, P.E. VILLAGRA \& J.N. ARANIBAR. 2010. Dinámica temporal de la masa de broza en distintos microhábitats del desierto del Monte Central (Mendoza, Argentina). Rev. Fac. Cienc. Agrar. 42: 55-69.

NEWTON, A.C. \& N. TEJEDOR. 2011. Principles and Practice of Forest Landscape Restoration: Case studies from the drylands of Latin America (p. xxvi + 383). UICN. Gland, Switzerland.

NOY-MEIR, I. 1973. Desert Ecosystems: Enviroment and Producers. Annu. Rev. Ecol. Syst. 4: 25-51.

PEROSA, M, J.F. ROJAS, P.E. VILLAGRA, M.F. TOGNELLI, R. CARRARA \& J.A. ALVAREZ. 2014. Distribución potencial de los bosques de Prosopis flexuosa en la Provincia Biogeográfica del Monte, desde Catamarca hasta Mendoza (Argentina). Ecología Austral 24: 238-248.

PASIECZNIK, N.M., P. FELKER, P.J.C. HARRIS, , L.N. HARSH, G. CRUZ, J.C. TEWARI, K. CADORET\& L.J. MALDONADO. 2001. The Prosopis juliflora - Prosopis pallida complex: a monograph. HDRA, Coventry. 


\section{J. A. Alvarez et al. - Bosques de Prosopis flexuosa de Catamarca}

PRIETO, M.R., P.E. VILLAGRA, N.B. LANA \& E.M. ABRAHAM. 2003. Utilización de documentos históricos en la reconstrucción de la vegetación de la Llanura de la Travesía (Argentina) a principios del siglo XIX. Rev. Chil. Hist. Nat. 76: 613-622.

PUGNAIRE, F.I., P. HAASE \& J. PUIGDEFABREGAS. 1996. Facilitation between higher plant species in a semiarid environment. Ecology 77: 1420-1426.

RICHARDS, L.A. 1954. Diagnóstico y rehabilitación de suelos salinos y sódicos. Manual de Agricultura $N^{o}$ 60. Departamento de Agricultura de los Estados Unidos de América.

ROIG-JUÑENT, S., G. FLORES, S. CLAVER, G. DEBANDI \& A. MARVALDI. 2001. Monte Desert (Argentina): insect biodiversity and natural areas. $J$. Arid Environ. 47: 77-94.

ROIG, F.A. 1993. Aportes a la etnobotánica del género Prosopis. In: IADIZA (ed.), Contribuciones Mendocinas a la Quinta Reunión de Regional para América Latina y el Caribe de la Red de Forestación del CIID. Conservación y Mejoramiento de Especies del Género Prosopis, pp. 99-119. Mendoza, Argentina.

ROIG, F.A., M.M. GONZÁLEZ LOYARTE, E.M. ABRAHAM, E. MENDEZ, V.G. ROIG, V.G. \& E. MARTÍNEZ CARRETERO. 1991. Maps of desertification hazard of Central Western Argentina (Mendoza Province) study case World Atlas of Thematic Indicators of Desertification. UNEP, London.

ROJAS, J.F. 2013. Procesos ambientales: deforestación y actividades productivas en los valles y bolsones del oeste de La Rioja y Catamarca desde mediados del siglo XIX. Tesis Doctoral. FFyL, UNCuyo, Mendoza

ROSSI, B.E., 2004. Flora y vegetación de la Reserva de Biosfera de Ñacuñán después de 25 años de clausura. Heterogeneidad espacial a distintas escalas. Tesis doctoral Universidad Nacional de Cuyo, Mendoza.

ROSTAGNO, C.M. \& H.F. DEL VALLE. 1988. Mounds associated with shrubs in aridic soils of northeastern Patagonia: characteristics and probable genesis. Catena 15: 347-359.

RUNDEL, P., P.E. VILLAGRA, M.O. DILLON, S.A. ROIG-JUÑENT \& G. DEBANDI. 2007. Arid and Semi-Arid Ecosystems. In: VEBLEN, T.T., K. YOUNG \& A. ORME (eds.), The physical geography of South America. pp. 158-183. Oxford University Press, Oxford.

ROSSI, B.E., VILLAGRA, P.E., 2003. Effects of Prosopis flexuosa on soil properties and the spatial pattern of understory species in arid Argentina. $J$. Veg. Sci. 14: 543-550.

SAyDS 2005. Primer Inventario Nacional de Bosques Nativos. Proyecto de Bosques Nativos y Áreas
Protegidas. Informe regional Bosque Andino Patagónico.

STOKES, M.A. \& T.L.SMILEY, 1968. And introduction to tree ring dating. University of Chicago Press, Chicago.

VÁZQUEZ, D.P., J.A. ALVAREZ, G. DEBANDI, J.N. ARANIBAR \& P.E.VILLAGRA. 2011. Ecological consequences of dead wood extraction in an arid ecosystem. Basic App Ecol 12: 722-732.

VILLALBA, R.1985. Xylem structure and cambial activity in Prosopis flexuosa D.C. IAWA Bull 6: 119-130.

VILLALBA, R., P.E. VILLAGRA, J.A. BONINSEGNA, M.S. MORALES \& V. MOYANO. 2000. Dendroecología y dendroclimatología con especies del género Prosopis. Multequina 9: 1-18.

VILLAGRA, P.E., M.A. CONY, N.G. MANTOVÁN, B.E. ROSSI, M.M. GONZÁLEZ LOYARTE, M.M., VILLALBA, R., MARONE, L., 2004. Ecología y Manejo de los algarrobales de la Provincia Fitogeográfica del Monte. In: ARTURI, M.F., FRANGI, J.L. \& J.F. GOYA (eds.), Ecología y Manejo de Bosques Nativos de Argentina. Editorial Universidad Nacional de La Plata.

VILLAGRA, P.E., CAVAGNARO, J.B., 2000. Effects of clayish and sandy soils on the growth of Prosopis argentina and $P$. alpataco seedlings. Ecología Austral 10, 111-119.

VILLAGRA, P.E., J.A. BONINSEGNA, J.A. ALVAREZ, M. CONY, E.M. CESCA \& R. VILLALBA. 2005. Dendroecology of Prosopis flexuosa woodlands in the Monte desert: Implications for their management. Dendrochronologia 22: 209-213.

VILLAGRA, P.E. \& R. VILLALBA. 2001. Estructura poblacional del algarrobal de la Reserva de Nacuñán. In: CLAVER, S. \& S. ROIG-JUÑENT (eds.), El desierto del Monte: La Reserva de Biósfera de Ñacuñán, pp. 71-75. IADIZA - MAB - UNESCO, Mendoza.

VILLAGRA, P.E., G. DEFOSSÉ, H. DEL VALLE, M.S. TABENI, C.M. ROSTAGNO, E. CESCA \& E.M. ABRAHAM. 2009. Land use and disturbance effects on the dynamics of natural ecosystems of the Monte Desert. Implications for their management. J. Arid Environ. 73: 202-211.

VILLAGRA, P.E., A. VILELA, C.V. GIORDANO, \& J.A. ALVAREZ, . 2010. Ecophysiology of Prosopis Species from the arid lands of Argentina: What do we know about adaptation to stressful environments? In: RAMAWAT, K.G. (ed.), Desert Plants. Biology and Biotechnology, pp. 322-354. Springer-Verlag, Berlin-Heidelberg.

VOLKMAR, K.M., Y. HU \&H. STEPPUHN, H., 1998. Physiological responses to plant salinity: a review. Can. J. Plant Sci. 78: 19-27. 
Bol. Soc. Argent. Bot. 50 (2) 2015

WALKLEY,A.I.B.1934. An examination of the Degtjareff method and a proposed modification of the chromic matter and a proposed modification of the chromic acid titration method. Soil Sci. 34: 29-38.

WHITFORD, W.G. 2002. Ecology of desert systems. Academic Press. San Diego, USA.

ZAR, J.H., 1984. Biostatistical analysis, Second edn. Prentice-Hall International Editions.

ZULOAGA, F.O. \& O. MORRONE (eds.). 1996. Catálogo de las plantas vasculares de la Argentina. I - Pteridophyta, Gimnospermae y Angiospermae (Monocotyledoneae). Monograph in Systematic Botany from the Missouri Botanical Garden, 60.
ZULOAGA, F.O. \& O. MORRONE (eds.). 1999. Catálogo de las plantas vasculares de la Argentina. II - Dicotyledoneae. Monograph in Systematic Botany from the Missoury Botanical Garden, 74.

Recibido el 8 de noviembre de 2014, aceptado el 17 de abril de 2015 . 